Instituto Internacional de Investigación y Desarrollo Tecnológico Educativo INDTEC, C.A.

DOI: https://doi.org/10.29394/scientific.issn.2542-2987.2017.2.3.8.155-175

OAI-PMH: http://www.indteca.com/ojs/index.php/Revista Scientific/oai

\title{
Alternativas para Transformar la Evaluación en la Praxis Universitaria
}

Autores: Carol del Carmen Terán González

Universidad de los Andes, ULA

carolterang@gmail.com

Trujillo, Venezuela

Wilmer José Araujo

Universidad Nacional Experimental Rafael María Baralt, UNERMB wilmeraraujo2017@gmail.com

Trujillo, Venezuela

Roexana Andreina Castillo Vázquez

Universidad de los Andes, ULA

roexanacastillo90@gmail.com

Trujillo, Venezuela

\section{Resumen}

Hablar de evaluación es un tema muy complejo ya que implica hacer una revisión del proceso de aprendizaje y como se ha aplicado en la realidad dicho concepto, principalmente en las instituciones universitarias, donde por lo general los profesores conocen como evaluación la calificación o nota que pueden asignar a sus alumnos para verificar los aprendizajes logrados. El objetivo del siguiente trabajo de investigación es Proponer alternativas para trasformar la evaluación de los aprendizajes en la praxis educativa universitaria. La investigación está enmarcada en investigación descriptiva, bajo la modalidad de proyecto factible. La propuesta tiene como objetivo principal Desarrollar alternativas para la transformación de la evaluación de los aprendizajes en la práctica universitaria. Para el diseño de la propuesta se plantean dos aspectos a considerar por el docente universitario basado en los instrumentos sugeridos al docente y las consideraciones acerca del enfoque constructivista a través de la evaluación cualitativa. En conclusión, se puede decir que los docentes de educación universitaria aplican un tipo de evaluación tradicional, de forma cuantitativa, olvidando que la evaluación es un elemento externo a la actividad de aprender.

Palabras clave: evaluación de los aprendizajes; evaluación cualitativa; praxis universitaria. 


\title{
Alternatives to Transform the Evaluation into the University Practice
}

\begin{abstract}
To speak about evaluation is a very complex topic since it implies making a deep analysis of the learning process one and since the above mentioned concept has been applied in the reality, principally in the university institutions, where in general the teachers know as evaluation the qualification or marks that they can assign to his/her pupils to check the successful learnings. The goal of this research is to propose some alternatives for transforming the evaluation of the learnings in the educational university practice. For the design of the investigation there appear two aspects to consider for the university teacher based on the instruments suggested to the teacher and the considerations that it brings over of the constructivist approach across the qualitative evaluation. In conclusion, it is possible to say that the teachers of university education apply a type of traditional evaluation, in a quantitative form, forgetting that the evaluation is an external element to the activity to learn.
\end{abstract}

Keywords: evaluation of learnings; qualitative evaluation; university practice. 


\section{A manera de Introducción}

La evaluación constituye uno de los elementos fundamentales en la práctica pedagógica, por lo que no es sorprendente que exista un número de estilos asociados a ella. Si solamente existiera un objetivo simple e inequívoco, la evaluación sería una cuestión mucho más sencilla de lo que es. La evaluación constituye parte importante en las universidades venezolanas, Alves y Acevedo (2002): la definen como una herramienta de valoración que busca comprender los hechos relacionados con el aprendizaje tal como suceden en la realidad de esta, un espacio para la reflexión, comprensión y valoración del desarrollo de los participantes. Es decir, la misma es una herramienta para encausar las experiencias a compartir en la praxis universitaria, centrando su interés en sus participantes como centros del proceso de aprendizaje.

En coherencia con este planteamiento, hoy día la evaluación orienta hacia el estudio de cada aspecto vinculado con los aprendizajes en la práctica universitaria y la búsqueda de la eficiencia académica como una forma de alcanzar una educación de calidad correspondiendo a las expectativas planteadas dentro del contexto universitario moderno.

Al respecto, Odreman (1996): lograr la calidad como filosofía en la educación, implica la necesidad de un cambio en las estrategias planteadas para el desarrollo de la práctica pedagógica, así como en la forma de valorar los resultados obtenidos. Por lo que, dentro de los cambios propuestos se incluirían todos los elementos pertenecientes al proceso de aprendizaje, señalando como uno de los principales la evaluación, por ser ella la que permite definir la eficiencia y eficacia de los procesos y estrategias aplicadas, además de los alcances que se dan en los participantes mediante la experiencia de aprendizaje compartidos en función de sus necesidades y expectativas.

La búsqueda de la excelencia en la Educación Universitaria es una 
exigencia actual que compromete a todos aquellos implicados en las tareas educativas y a todos los ámbitos, facetas y componentes del proceso de formación de los futuros profesionales. En el país, esa búsqueda se manifiesta en la voluntad de un perfeccionamiento continuo de la enseñanza universitaria. En relación a lo antes dicho, la evaluación educativa y en particular, la del aprendizaje de los estudiantes, constituye un contexto un tema de singular interés, por su importancia, complejidad y por la vigencia de numerosos problemas pendientes de solución.

Al respecto, Martínez, Gil y Martínez (2003: 242): mencionan que el resultado suele ser la creación de una circularidad en que se enseña y se estudia para los exámenes, convirtiéndose la evaluación en la dueña de la enseñanza y el aprendizaje, en vez de ser un instrumento que impulse a aprender y enseñar mejor. Hablar de evaluación es un tema complejo que implica hacer una revisión del proceso de aprendizaje y como se ha aplicado en la realidad dicho concepto, principalmente en las instituciones universitarias, donde por lo general los profesores conocen como evaluación la calificación o nota que pueden asignar a sus alumnos para verificar los aprendizajes logrados. Los estudiantes por su parte, relacionan la evaluación con presentar un examen, pasar una materia y obtener una calificación; todo esto va acompañado de sentimientos negativos hacia al proceso de evaluación.

Con respecto a lo mencionado anteriormente, los docentes Venezolanos deberían estar en condiciones, no solo de elegir, sino de generar alternativas de evaluación dentro de un enfoque del proceso educativo. Por estas razones, es posible trasformar la evaluación en una herramienta de conocimiento para los profesores y alumnos; aquí surge la necesidad de que el docente sea responsable de su elaboración didáctica, debe ser a su vez libre de diseñar y administrar su función, más que la de servir de control, minimizando sus posibles efectos positivos de mejoramiento de los procesos 
y de los resultados.

Por lo antes señalado, hay que mirar la evaluación en Venezuela desde una perspectiva holística e integral, donde no solamente se tome lo cuantitativo, sino también otros aspectos del educando como la sus cualidades para realizar una evaluación descriptiva del proceso de aprendizaje integral del alumno; además se debe tener en cuenta, los conocimientos previos de los educandos, su actuación en el aula, las evaluaciones continuas, los factores extra instituciones, tales como: los intereses, motivaciones, las características socio económica, el entorno social y familiar en el que se desenvuelven los estudiantes.

Se parte así de la concepción de la evaluación como un proceso sistemático integral, gradual y continuo que valora los cambios producidos, eficacia, técnicas de capacidad del educador; esto con la finalidad de convertirse en un proceso de mejora continua por medio de la pro alimentación, satisfacción de los conocimientos adquiridos, toma de decisiones, acciones entre otras cosas.

La evaluación se puede entender como el proceso mediante el cual se emite a un juicio de respeto de uno o más atributos de algo o alguien fundamentado en formación obtenida procesada y analizada correctamente y contrastada con un referente claramente establecido en un marco de referencia valórico y consistente con el que está encaminado a mejorar los procesos educacionales y que produce efectos sobre sus participantes, para lo que se apoya en el diálogo y compresión (Zabalza, 2002: 28).

La evaluación es aquella donde se juzga o valora más la calidad tanto del proceso como del nivel de aprovechamiento alcanzado de los alumnos como resultado de la dinámica del proceso de enseñanza aprendizaje. La misma procura lograr una descripción holística, esto es que intenta analizar exhaustivamente, con sumo detalle, tanto la actividad como los medios y el aprovechamiento alcanzado por los alumnos en la sala de clase. Sin embargo, 
nadie puede negar que la evaluación cualitativa, sea una actividad compleja, pero al mismo tiempo constituye una tarea necesaria y fundamental en la labor docente, así lo afirma Morán (2007): cuando dice la evaluación cualitativa es aquella donde se juzga o valora más la calidad del proceso como el nivel de aprovechamiento alcanzado por los alumnos que resulta de la dinámica del proceso de enseñanza aprendizaje.

Así mismo, se puede señalar que el docente al desempeñar sus funciones dentro de la praxis universitaria, debe tener cierta concepción explícita del modo en que se enseña y se aprende con la evaluación cualitativa, sobre qué, cómo, cuándo, por qué y para qué se va a evaluar, con el fin de asegurar que las experiencias educativas que se propongan en el acto de enseñanza produzcan resultados positivos. El docente en su desempeño laboral, debe poseer ciertos conocimientos y prácticas precisas de todo un nutrido arsenal de instrumentos y técnicas para evaluar cualitativamente los aprendizajes de los alumnos, en momentos pertinentes en que decida hacerlo, sea porque él lo considere o la institución o el currículo se lo demanden.

Por lo antes dicho, la evaluación que se realiza por parte de los docentes en el ámbito de la escuela básica, pasando por el bachillerato hasta llegar a la universidad es una evaluación de los aprendizajes, la cual consiste en un proceso de valoración continua, que permite recoger y analizar evidencias sobre: experiencias previas del alumno, su desarrollo evolutivo, alcances progresivos en relación con las competencias básicas de grado derivadas de los objetos generales de la etapa, así como las condiciones en las cuales se realiza el aprendizaje para propiciar la toma de decisiones consensuadas entre los participantes, a fin de orientar retroalimentar y mejorar el proceso de enseñanza-aprendizaje. Es importante señalar que, la medición y evaluación del aprovechamiento académico no es sólo una tarea intelectual que se suele medir únicamente con los exámenes; también depende de la conducta del educando en términos de actitud, intereses, sentimientos, 
carácter y otros atributos de la personalidad.

En la práctica universitaria, para el facilitador no es fácil juzgar la calidad de los aprendizajes de sus alumnos al tener que considerar estos como parte integral de su comportamiento respecto a las diversas dimensiones de la conducta humana por su condición subjetiva e intangible, ya que el aprendizaje requiere medios y técnicas especializadas. El proceso de evaluación no concluye hasta que se haya transmitido la retroalimentación de la interpretación de los desempeños de sus alumnos. Esta retroinformación sirve para que el alumno pueda detectar sus progresos o dificultades y el docente programe nuevas situaciones de aprendizaje con el propósito de equilibrar los desempeños esperados con los producidos.

Desde la perspectiva antes señalada, el planteamiento del tema de la evaluación del aprendizaje del alumno en la universidad venezolana se sitúa como una problemática compleja, ya que no solo se acerca a dimensiones no meramente técnicas, sino también afectivas y organizadoras, ideológicas, entre otras. No obstante, la propuesta para afrontar la problemática consiste en plantearla, aunque de ello se derive más inquietud que tranquilidad. Sin duda, pocas tareas provocan tantas dudas y contradicciones a los docentes como las relacionadas con la evaluación y las actuaciones o decisiones asociadas a ella. Además, esta evaluación trasciende el ámbito puramente académico y se traslada a la propia sociedad, que acoge, a veces entre los elementos de éxito o fracaso la propia evaluación que reciban sus ciudadanos.

Ante lo expuesto se deben tomar en cuenta los conocimientos previos de los educandos, su actuación en el aula, las evaluaciones continuas, los factores extra que forman parte del hecho educativo; por estas razones, surge la siguiente interrogante: ¿Qué alternativas puede poner en práctica el docente para transformar la evaluación de los aprendizajes en la praxis universitaria? 


\subsection{Propósito de la investigación}

Proponer alternativas para trasformar la evaluación de los aprendizajes en la praxis educativa universitaria.

\section{Recorrido teórico}

\subsection{En búsqueda de la evaluación en la praxis universitaria}

Una buena evaluación debe tratar de describir (lo que está bajo discusión), valorar y remediar (los errores y deficiencias). La evaluación tradicional normalmente es válida sólo en el segundo aspecto (valorar), y con frecuencia se olvida del tipo de consejo y apoyo que necesitan los estudiantes para triunfar en sus estudios (Brown y Glasner, 2003: 37). Otro aspecto negativo de la evaluación tradicional, es que con frecuencia se evalúa en el momento temporal que conviene más al sistema (programación) o al profesor a nivel individual que cuando es más conveniente para el desarrollo integral del alumno. Los sistemas tradicionales tampoco son adecuados para poder remediar los errores y deficiencias de los alumnos, ya que no existe tal posibilidad si nos basamos en un único examen al final del curso.

En general, un sistema de evaluación continua debería basarse en una "evaluación formativa", ya que la "evaluación sumativa" se basa en un punto final que es numérico y que encierra juicios evaluativos. Sin embargo, en la práctica se presenta un grave problema: las instituciones universitarias acaban exigiendo una graduación en las calificaciones $y$, por lo tanto, obligan a efectuar evaluación sumativa. Observando el siguiente cuadro en cuanto a la innovación de la evaluación.

Los niveles referidos son: a). Los objetivos o competencias que se aspiran de la innovación, b). El nivel de los criterios o patrones con los que se comparará la situación novedosa para su comprobación y c). El nivel de la correspondencia que guarda el nuevo procedimiento con un cambio real.

La evaluación como proceso de aprendizaje y, a la vez, como 
herramienta de retroalimentación al proceso en la práctica universitaria, tiene una serie de características que le confieren una función de mejora, de información, de retroalimentación, de orientación, de posibilidad de aprendizaje continuo, de ayuda y asesoramiento. Éstas son:

- Holística: el proceso se antepone al resultado.

- Democrática: la participación de todos los que intervienen en el proceso de evaluación es fundamental.

- Ética: parte del explicitación de criterios que remiten a valores, intereses y expectativas.

- Política e ideológica: responde a una forma de concebir el proceso de aprendizaje. No es una actividad técnica.

- Busca la equidad: no se queda anclada en una objetividad que deshecha los procesos y las vivencias de las personas, por lo que utiliza como procedimientos habituales la autoevaluación y la coevaluación.

- Justa: busca la justicia desde una dimensión valorativa de las cosas.

- Crítica: ya que genera aprendizajes para docentes y estudiantes.

- Dinamizadora de la práctica: porque propone criterios para la innovación y la mejora de la práctica docente.

- Formativa: o educativa al buscar la calidad de la enseñanza.

- Continúa: al establecer un proceso permanente en el tiempo que asegura el éxito de los alumnos en la consolidación de los objetivos de aprendizaje planteados.

- Integradora, individualizada y personalizada.

- Fomenta el desarrollo de capacidades, teniendo en cuenta a la persona y al contexto. Margalef, L. (2007: 9).

Autores como Álvarez y Méndez (2001: 25), sostienen que la evaluación como proceso de aprendizaje y, a la vez, como herramienta de retroalimentación al proceso en la práctica universitaria, tiene una serie de características que le confieren una función de mejora, de información, de retroalimentación, de orientación. Mientras que Brown y Glasner (2003): defienden la evaluación como actividad crítica de aprendizaje, como promotora de la comprensión, y que contribuye a la "emancipación de los estudiantes, a su desarrollo personal, moral, académico y social." (Margalef, L. 2007). En este sentido, comprendiendo la evaluación como aprendizaje, se entiende que se debe ir en búsqueda de desarrollar y por tanto, evaluar habilidades 
intelectuales relacionadas con la comprensión, la aplicación y el razonamiento (comparación, relación de ideas, argumentación, proposición, contrastación, la reconstrucción del sentido y del significado a partir de la información).

Autores como Brown y Glasner (2003: 101): proponen algunas indicaciones para realizar una evaluación alternativa teniendo en cuenta que ésta implica la utilización de nuevas estrategias de evaluación, alternativas o diferentes al ya conocido "examen":

a. Considerar cuidadosamente la carga de trabajo del estudiante: ¿Cuánto tiempo lleva al estudiante trabajar sobre estrategias de evaluación alternativas?

b. Adoptar medidas para mantener el interés: calendarios que expliciten las fechas de entregas de trabajos, retroalimentación constante, permanente orientación al estudiante.

c. Ser precavidos al introducir una nueva forma de evaluación: el estudiante puede tener reticencias hacia la nueva forma de evaluación, por lo que es muy necesario explicar el porqué de la forma de evaluar y analizar con ellos la importancia que tiene para su propio proceso de aprendizaje.

d. Establecer marcos de actuación claros.

e. Orientar sobre los criterios de evaluación a ser utilizados: de manera que no queden dudas y que este proceso permita la apropiación de los criterios por parte del estudiante.

\subsection{Autoevaluación, coevaluación y realimentación.}

Al hablar sobre los propósitos de la evaluación se hace referencia al papel fundamental de la evaluación como orientación al docente, al estudiante, a los planificadores y admiradores de la educación. De esta función se desprenden procesos que son inherentes al proceso de evaluación, estos son la Autoevaluación, Coevaluación y realimentación. Es importante señalar que la debe tratar de describir (lo que está bajo discusión), valorar y remediar (los errores y deficiencias). La evaluación tradicional normalmente es válida sólo en el segundo aspecto (valorar), y con frecuencia se olvida del tipo de consejo y apoyo que necesitan los estudiantes para triunfar en sus estudios.

Se ha de insistir que los procedimientos de evaluación adquieren un 
sentido u otro, se aplican de una u otra manera, según la actitud con la que se aborda la actividad evaluativa. Algunos términos como diálogo, consenso, flexibilidad, autorreflexión, coevaluación y participación deben animar la actividad evaluativa si se pretende lograr una verdadera transformación en la praxis evaluativa universitaria y que tenga un impacto en la calidad de los procesos de aprendizaje de los participantes en el hecho educativo.

Autoevaluación, para concretar la definición de autoevaluación debe remitirse a la idea fundamental de la de aprendizaje. A este respecto debe comprenderse que, el mismo se produce por una reacción del individuo en respuesta al medio ambiente. Es decir, es la respuesta que da el individuo a las experiencias que obtiene del medio.

Coevaluación: Esta alude a las secciones de clase en las que, con la participación o no del facilitador, se realiza un esfuerzo por evaluar la efectividad del grupo completo o de algunos subgrupos en cuanto a ciertos fines. La coevaluación es producto de la comunidad de aprendizaje, por tanto, son los mismos alumnos y alumnas quienes valoran sus progresos y debilidades. El docente, en este caso, solo cumple la función de orientadora de los juicios emitidos, evitando que los mismos trasgredan las pautas de valoración establecidas.

Realimentación: El proceso de realimentación tiene dos componentes básicos: evaluación y comunicación. El profesor realiza en primer lugar una evaluación al estudiante y a continuación le comunica los resultados obtenidos (aciertos y errores) en la operación anterior. Las razones por las cuales la retroalimentación es indispensable son; primero que es difícil mejorar lo que está haciendo, a menos que se conozcan los resultados de lo que se está realizando. Sin este conocimiento se tendría a repetir errores y con ello a reforzarlos, lo cual es contrario a la noción de aprendizaje. 


\section{Metodología Empleada}

Se realizó la investigación desde un enfoque descriptivo bajo a la modalidad de los proyectos factibles, según el componente docente de la Universidad Pedagógica Experimental Libertador (UPEL, 2002: 07): define el "proyecto factible "como estudio sistemático" que consiste en la investigación, elaboración y desarrollo de una propuesta de un modelo operativo viable para solucionar problemas, requerimientos o necesidades de organizaciones 0 grupos sociales". Así lo afirma Hurtado (2000: 130): la investigación proyectiva consiste en encontrar la solución a los problemas prácticos, se ocupa de cómo deberían ser las cosas para alcanzar los fines y funcionar adecuadamente. Por lo que, esta investigación tiene como objetivo general Proponer alternativas que permitan la transformación de la evaluación de los aprendizajes en la praxis educativa. Se basa en el apoyo que orienta la investigación sobre el sustento minucioso de un diseño documental y de campo, el diseño de la investigación es la estrategia general que adopta el investigador para dar respuesta a la problemática planteada Es importante señalar que para la recolección de datos se aplicó un cuestionario semiestructurado.

En este sentido, la investigación descriptiva como toda investigación, ha de tener por norte satisfacer una o varias necesidades de modo que permita obtener los datos, resolver un tipo de situación-problema o en todo caso recoger elementos para actuar sobre este. De lo anterior, se deduce que aunque el objeto de investigación es llegar a conocer aptitudes predominantes en la descripción exacta de las actividades, es bueno advertir que no se encuentra limitada a la simple recolección de datos, sino que también puede predecir, identificar y proponer alternativas de solución cuando la investigación la amerite y el investigador lo considere apropiado como es el caso de la misma que tiene como objetivo proponer estrategias al docente para evaluación cualitativa en la praxis universitaria. 


\section{Propuesta}

Para el diseño de la propuesta, se plantean dos aspectos a considerar por el docente universitario basado en los instrumentos sugeridos a este y las consideraciones acerca del enfoque constructivista, a través de la evaluación cualitativa, los cuales se describirán a continuación.

\subsection{Instrumentos de evaluación a considerar como sugerencia al docente.}

Al mencionar la evaluación de los aprendizajes en las últimas décadas, el concepto de evaluación ha sufrido una profunda transformación significativa en el ámbito de la enseñanza y del aprendizaje, ha sido un elemento externo a la actividad de aprender. Se le ha considerado y se le considera, tanto desde las perspectivas cualitativas como cuantitativas, como un medio por el que se valora un aprendizaje, y a partir de los datos obtenidos, se inician nuevos aprendizajes.

Hoy el aprendizaje y la evaluación deben tomar en consideración el desarrollo del propio estudiante, es decir, sus expectativas, su nivel inicial, sus estilos de aprendizaje, sus ritmos e intereses, sus necesidades y proyección futura. Desde esta perspectiva, el reto de la evaluación es cómo debe plantearse para ser congruente con las teorías que se propugnan para un aprendizaje significativo y respetuoso con las peculiaridades individuales y culturales del alumnado y sus necesidades. De allí, el uso de instrumentos evaluativos como el portafolio, foto texto, las construcciones mentales (mapas conceptuales y mentales) contextualizaciones de trabajos monográficos y papeles ensayísticos basados en su realidad.

El portafolio es una colección selectiva deliberada y variada de los trabajos del estudiante donde se reflejan sus esfuerzos, progresos y logros en un período de tiempo y en alguna área específica. (Villarini, 1996): al desarrollar esta estrategia proyecta la diversidad de aprendizajes que ha 
interiorizado. En este modelo se detectan los aprendizajes positivos, las situaciones problema, las estrategias utilizadas en la ejecución de tareas. La estrategia portafolio es considerada tanto una técnica de enseñanza aprendizaje de la autoevaluación, como una forma de evaluación alternativa.

Fischer y King (1995: 69): se refieren a él como a una tarea multifacética que supone diversos tipos de actividades y cuya realización se efectúa en un período de tiempo. Farr y Tone (1994: 78): consideran que el portafolio contiene un conjunto de pensamientos, ideas y relaciones que permiten dirigir el desarrollo del aprendizaje del alumnado. Beckeley (1997): añade que tiene un carácter cooperativo, ya que implica a participantes y docentes en la a organización y desarrollo de su propia evaluación. El portafolio está dirigido a la práctica diaria académica y puede contemplar, además, aprendizajes indirectos de formación. La determinación de los materiales se efectúa con unos criterios de selección, de evaluación y de validez y ha de estar organizado (tipología de estrategia, unidades de contenidos de forma mixta), considerando una amplia diversidad de tareas.

Por estas razones, en el proceso de realización del portafolios siempre ha de estar presente el balance entre el proceso y el producto de aprendizaje, la evidencia del progreso y del desarrollo del alumno y de la alumna, y una amplia variedad de tareas y materiales referidas a diversas competencias, estrategias y habilidades del alumnado (en función del contenido del período al que corresponde), a su vez, el análisis reflexivo de los resultados parciales y globales, es decir, el aprendizaje al mismo tiempo de la autoevaluación válida, en un contexto.

\subsection{Consideraciones para un acercamiento a la transformación de la evaluación desde un enfoque cualitativo-constructivista.}

Para el desarrollo de un enfoque constructivista donde el participante y el facilitador construyan alternativas cualitativas que favorezcan la 
transformación de la evaluación en la práctica universitaria, la evaluación ha de ser entendida como un proceso que promueve el aprendizaje y no como un control externo realizado por el profesorado sobre lo que hace el alumno y cómo lo hace.

La evaluación, incluida en el mismo acto de aprendizaje comporta una mayor comprensión tanto por parte del profesor como del estudiante de los procesos que se están realizando, así como el conocimiento de las razones de los errores y aciertos que se producen. El acto evaluativo, desde esta perspectiva, más que un proceso para certificar o aprobar, se coloca como participante, como optimizador de los aprendizajes contribuyendo a proporcionar información relevante para introducir cambios y modificaciones para hacer mejor lo que se está haciendo.

La evaluación, además, pasa a ser un elemento vivo con una causalidad y una aportación para el alumno. Evaluar no es "demostrar" sino "perfeccionar" y "reflexionar". La evaluación debería convertirse en un proceso reflexivo donde el que aprende toma conciencia de sí mismo y de sus metas y el que enseña se convierte en guía que orienta hacia el logro de los aprendizajes. Por ello se deben tomar en cuenta los siguientes aspectos:

- Promover acciones evaluativas que ponga en juego la significatividad de los nuevos aprendizajes a través de su uso en la resolución de problemas, aplicación a distintos contextos, en la construcción de nuevos conocimientos.

- Evitar los modelos memorísticos en los que sólo se pone de manifiesto la capacidad para reconocer o evocar.

- Construir actividades y tareas de evaluación que tengan sentido para el alumnado.

- Utilizar una gama variada de actividades de evaluación que ponga en funcionamiento los contenidos en contextos particulares diversos. Lo importante es contextualizar.

- Evaluar el mismo contenido con distintas técnicas: una actividad de evaluación es parcial en cuanto a la naturaleza y amplitud de relaciones del significado que explora, es previsible que el alumno y la alumna disponga de otras relaciones significativas que el instrumento o procedimiento de evaluación que se utiliza no logra alcanzar.

- Promover distintas maneras de evaluación y tareas alternativas donde 
el estudiante pueda elegir.

- Utilizar procedimientos que permitan al estudiante aprender a construir su forma personal de realizar el aprendizaje, a manejar autonómicamente procedimientos de evaluación y corregir los errores que pueda detectar.

- Motivar al estudiante para que haga suyo los objetivos del aprendizaje y los criterios que se van a utilizar para evaluarlos.

- Fomentar la autoevaluación, que el estudiante piense acerca de cuánto aprende sus fortalezas y debilidades de forma crítica y reflexiva.

- Estimular procesos de co-evaluación entre el facilitador y el participante.

- Presentar en las evaluaciones situaciones lo más parecida posible a la realidad.

- Incluir tareas de manera natural durante el proceso de evaluación y de enseñanza aprendizaje que puedan servir al estudiante para tomar conciencia de lo que han aprendido y de las dificultades o lagunas que todavía tiene.

\section{Conclusiones}

Una vez culminada la presente investigación titulada Alternativas para transformar la evaluación en la praxis universitaria, tomando en cuenta los resultados obtenidos en cuanto a los objetivos planteados en ella, se llegó a la siguiente conclusión:

El docente universitario manifiesta mayor disposición para el uso de instrumento como pruebas orales y escritas al momento de evaluar como recurso, a fin de determinar el alcance del aprendizaje de los participantes en el aula; dejando a un lado recursos como los mapas mentales, el portafolio; sin embargo, un número significativo de educadores emplea las minutas y los ensayos.

Así mismo, se determinó que la mayoría de educadores en la praxis universitaria prefieren el trabajo individual por parte de los estudiantes que el trabajo colaborativo. En este sentido, al momento de evaluar no se aplica la Heteroevaluación, así como también se deja a un lado la autoevaluación y la coevaluación, las cuales son parte importante dentro del proceso de evaluación del aprendizaje. Por esta razón, es de hacer mención que los profesores no fomentan en sus estudiantes la responsabilidad de evaluarse, 
de reflexionar acerca de las debilidades y fortalezas que desarrollaron durante el desarrollo del proceso de enseñanza aprendizaje.

En concordancia con lo evidenciado anteriormente, se puede decir que los docentes de educación superior trabajan con un tipo de evaluación tradicional, de forma cuantitativa, olvidando que la evaluación ha sido un elemento externo a la actividad de aprender. Se la ha considerado y se la considera, tanto desde las perspectivas cualitativas como cuantitativas, como un medio por el que se valora un aprendizaje y, a partir de los datos obtenidos, se inician nuevos aprendizajes o, si es necesario, se realizan actividades de recuperación.

Se ha de insistir que los procedimientos de evaluación adquieren un sentido u otro, se aplican de una u otra manera, según la actitud con la que se aborda la actividad evaluativa. Algunos términos como diálogo, consenso, flexibilidad, autorreflexión, coevaluación y participación deben animar la actividad evaluativa si se pretende lograr una verdadera transformación en la praxis evaluativa universitaria y que tenga un impacto en la calidad de los procesos de aprendizaje de los participantes en el hecho educativo.

\section{Referencias}

Alves, E., y Acevedo, R. (2002). Evaluación Cualitativa. Reflexión para la transformación de la realidad educativa. Segunda Edición. Caracas: Petroglifos Producciones.

Álvarez y Méndez, J. (2001). Evaluar para conocer, examinar para excluir. Editorial Morata. Madrid.

Beckeley, W. (1997). Creating a classroom portfolio system. A guide to assist classroom teachers in kindergarten through eighth grade. lowa, Kendall/Hunt Publishing Company.

Brown, S. y Glasner, A. (2003). Evaluar en la universidad. Problemas y nuevos enfoques. Editorial Nancea. Madrid. 
Farr, R., y Tone, B. (1994). Portfolio and performance assessment. Helping students evaluate their progress as readers and writers. Fort Worth: Harcourt Brace College Publishers.

Fischer, Ch., y King, R. (1995). Authentic Assessment. A guide to implementation. Thousand Oaks, CA: Corwin Press, Inc.

Hurtado, J. (2000). Metodología de la Investigación Holística. Editorial Sypal. Caracas, Venezuela.

Margalef, L. (2007). El proceso de evaluación: estrategias, procedimientos y criterios. Vicerrectorado de Planificación Académica y profesorado. Dirección de Formación del profesorado Universitario. Universidad de Alcalá. Madrid.

Martínez, J., Gil, D., y Martínez, B. (2003). La universidad como nivel privilegiado para un aprendizaje como investigación orientada. En Monereo, C. y Poco, J. I. (eds). La universidad ante la nueva cultura educativa.

Morán, P. (2007). Hacia una evaluación cualitativa en el aula. Reencuentro, (48), 9-19, e-ISSN: 0188-168X. México: Universidad Autónoma Metropolitana Unidad Xochimilco.

Odreman, N. (1996). Proyecto Educativo. Educación Básica: reto compromiso y transformación. Caracas: Ministerio de Educación. Mimeografiado.

Zabalza, M. (2002). Innovación y cambio en los centros educativos. Micat. Santiago de Compostela.

UPEL (2002). Manual: Proyectos Factibles. Carcas: Universidad Pedagógica Experimental Libertador.

Villarini, A. (1996). 1er. Seminario taller sobre fundamentos y principios de evaluación auténtica. República Dominicana: Facultad Autónoma de Santo Domingo. 


\section{Carol del Carmen Terán González}

e-mail: carolterang@gmail.com

Nacida en la ciudad de Valera Edo Trujillo, Venezuela.

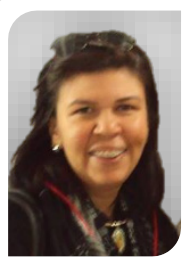
Cursó estudios de Doctorado en Educación en la Universidad Rafael María Baralt, posee Maestría en Docencia para la Educación Superior en la misma universidad, Maestría en Literatura Latinoamericana en la Universidad de los Andes y Licenciatura en Educación Mención Castellano y Literatura ULA-NURR investigadora del Centro de investigaciones literarias y lingüísticas Mario Briceño Iragorry. (CILL) ULANURR, Jefe de la unidad de investigación y proyecto de la Casa de Historia Trujillo, articulista e investigadora con 14 años de servicio en el área de educación y literatura. Ponente en eventos nacionales e internacionales. Docente invitada en pregrado y Postgrado, ULA, UNERMB, UNESR. Coordinadora de la Línea de investigación Memoria, Educación y Discursos emergentes (UNERMB), miembro de la Línea de investigaciones Ciudadanía, hermenéutica y proyectos sociales. (UNERS). Ha realizado artículos en distintas revistas arbitradas del país entre ella Sapiencia, Cifra Nueva, Revista de Cultura Centro Nacional de Historia, Revista de Ingeniería UVM, entre otras. 


\section{Wilmer José Araujo}

e-mail: wilmeraraujo2017@gmail.com

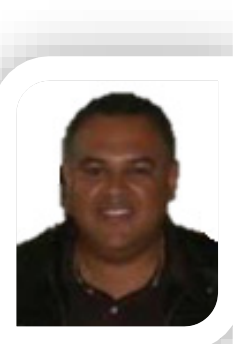

Nacido en la ciudad de Valera Edo Trujillo, Venezuela.

Doctorante en Educación Universidad Rafael María

Baralt, Maestría en Docencia para la Educación

Superior en la misma universidad, Licenciado en

Educación Mención Química ULA-NURR. Docente de Media General del Ministerio de Educación con 17 años de servicio. Docente invitado postgrado UNERMB. Desempeñó funciones como docente de pregrado en la Universidad Alonso de Ojeda. Miembro de la Línea de investigación Memoria, Educación y Discursos emergentes (UNERMB). 


\section{Roexana Andreina Castillo Vázquez}

e-mail: roexanacastillo90@gmail.com

Nacida en Valera, estado Trujillo, Venezuela.

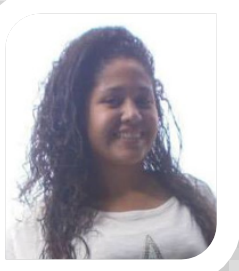

Estudiante de educación mención Lenguas Extranjeras del Núcleo Universitario "Rafael Rangel" ULA. Fue preparadora en el área de francés por dos (2) años. Ha participado en numerosos eventos de investigación en distintas áreas como ponente, comité organizador y asistente. Adscrita al Centro de Investigaciones Lingüísticas y Literarias "Mario Briceño Iragorry" ULA-NURR. Miembro de la línea de investigación Memoria, Educación y Discursos emergentes (UNERMB). Miembro del Taller "AbyaYala" NURR.

El contenido de este manuscrito se difunde bajo una Licencia de Creative Commons ReconocimientoNoComercial-Compartirlgual 4.0 Internacional 